\title{
Epigallocatechin gallate inhibits the proliferation of colorectal cancer cells by regulating Notch signaling
}

This article was published in the following Dove Press journal:

OncoTargets and Therapy

7 March 2013

Number of times this article has been viewed

\section{Heiying Jin ${ }^{1, *}$ \\ Wei Gong,** \\ Chunxia Zhangl,* \\ Shuiming Wang'}

'National Center of Colorectal Surgery, the Third Affiliated Hospital of Nanjing University of Traditional Chinese Medicine, Nanjing, People's Republic of China; ${ }^{2}$ Department of Surgery, Jiangyin Hospital of Traditional Chinese Medicine, Jiangsu, People's Republic of China

*These authors contributed equally to this work
Correspondence: Heiying Jin National Center of Colorectal Surgery, the Third Affiliated Hospital of Nanjing University of Traditional Chinese Medicine, I Jinling Road, Nanjing, 210001, People's Republic of China Fax +86 2552276200 Email jinheiying@yahoo.com.cn
Aims: To explore the inhibitory effects of epigallocatechin gallate (EGCG) on the proliferation of colorectal cancer cells and on the gene expression of Notch signaling.

Methods: The colorectal cancer cells and orthotopic colorectal cancer transplant model were treated with EGCG, and MTT assay was used to test the inhibitory role of EGCG in the proliferation of colorectal cancer cells.

Results: MTT assay indicated that EGCG inhibited the proliferation of these four cell lines when the time and concentration increased, and EGCG enhanced the apoptotic rate of these four cell lines. The dosage was positively correlated to the apoptotic rate, and EGCG inhibited the proliferation of colorectal cancer cells by influencing cell cycle. In-vivo study suggested that on the seventh day, the volume of tumors reduced after administrating with 5,10 and $20 \mathrm{mg} / \mathrm{kg}$ of EGCG. At the twenty-eighth day, the volume of tumors was significantly different in three EGCG treatment groups as compared to the control group $(P<0.05)$, and TUNEL assay indicated that the apoptosis of cancer cells in EGCG treated groups was markedly higher than that in the control group $(P<0.05)$. In these cell lines, the expressions of HES1 and Notch2 in EGCG treated groups were remarkably lower than that in the control group $(P<0.05)$. The expression of $J A G 1$ decreased in SW480 cells $(P=0.019)$, HT-29 cells and HCT-8 cells, but increased in LoVo cells at mRNA level. The expression of Notch1 was upregulated in these four cell lines, but its expression was significantly upregulated only in LoVo and SW480 cells $(P<0.05)$.

Conclusion: In-vitro and in-vivo studies showed that EGCG inhibited the proliferation, induced the apoptosis and affected the cell cycle of colorectal cancer cells. After treating with EGCG, the expressions of HES1 and Notch2 was obviously inhibited, this indicated that EGCG inhibited colorectal cancer by inhibiting HES1 and Notch2.

Keywords: EGCG; Colorectal cancer cells; Cell apoptosis; Cell cycle; Notch signaling

\section{Introduction}

Green tea is a conventional drink that is a standard beverage in Asia. Epidemiological investigations and experimental studies have shown that green tea and its extracts play an important role in the chemical prevention and treatment of many tumors, with no untoward side effects observed in normal tissue. ${ }^{1}$ Tea polyphenol is one of the main components of green tea; it affects the development of tumors in many ways, such as the growth inhibition of tumor cells, the prevention of mutations, apoptosis and differentiation of tumor cells, as well as the inhibition of angiogenesis. ${ }^{2}$ Epigallocatechin Gallate (EGCG) is a tea polyphenol monomer of the highest quantity and strongest activity; it inhibits tumors located in the skin, lungs, mouth, esophagus, stomach, small intestine, colon, bladder, and prostate. ${ }^{3}$ Previous studies have suggested that EGCG plays its inhibitory role in colorectal cancer by regulating the cell cycle, inducing 
apoptosis of cells and the inhibition of many different signaling pathways. ${ }^{4-6}$

Recent studies have indicated that stem cells associated with colorectal cancer play an important role in the development of this cancer as well its metastasis. In addition, Notch signaling plays a pivotal role in regulating the development, renewal, and maturation of tumor stem cells. However, whether these processes are regulated by EGCG remains unknown. In this study, the role of EGCG in colorectal cancer was systematically studied using both in-vitro and in-vivo experiments. In addition, the gene expression associated with Notch signaling was analyzed to better understand the pathways involved in inhibiting colorectal cancer by EGCG. The findings provide a potential novel method that might be used for prevention and therapy of colorectal cancer at the stem cell level.

\section{Materials and methods MTT}

3-(4,5)-dimethylthiazol (-z-y1)-3,5-diphenytetrazoliumromide (MTT) assay was used. LoVo, SW480, HCT-8, and HT29 colorectal cancer cell lines and EGCG were purchased from the Institute of Biochemistry and Cell Biology, Chinese Academy of Sciences (Shanghai, People's Republic of China). As per a previous study, ${ }^{3}$ LoVo, SW480, HCT-8, and HT-29 cells were seeded in 96-well plates at a concentration of $5 \times 10^{3}$ cells; each cell line was totally seeded in the 12 wells. Complete medium was added to the wells, up to $200 \mu \mathrm{L}$; the medium contained $0 \mu \mathrm{g} / \mathrm{mL}, 10 \mu \mathrm{g} / \mathrm{mL}, 20 \mu \mathrm{g} / \mathrm{mL}$, and $35 \mu \mathrm{g} /$ $\mathrm{mL}$ of EGCG. The inhibition rate $=[1-($ absorbance of EGCG group - absorbance of control group)/(absorbance of control group - absorbance of blank control group)] $\times 100$.

\section{Flow cytometry for the detection of apoptosis and cell cycle stages}

Flow cytometry detects apoptosis as reported previously. ${ }^{3}$ A total of $0.5 \times 10^{6}$ resuspended cells were centrifuged at $1000 \mathrm{~g}$ for 5 minutes and were then resuspended using $195 \mu \mathrm{L}$ Annexin V-fluorescein isothiocyanate (FITC) binding solution. An additional $5 \mu \mathrm{L}$ Annexin V-FITC was added to the tube and the mixture was gently mixed under photophobic conditions and incubated at room temperature for 10 minutes. The cells were further centrifuged at $1000 \mathrm{~g}$ for 5 minutes and resuspended using $190 \mu \mathrm{L}$ Annexin V-FITC binding solution. $10 \mu \mathrm{L}$ propidium iodide was added into the solution and gently mixed. Immediately following this step, flow cytometry was performed. The
Annexin V-FITC showed a green fluorescence and the propidium iodide showed a red fluorescence. The cell cycle was studied by flow cytometry, and the data was analyzed using ModFit LT ${ }^{\mathrm{TM}}$ (Verity Software House, Topsham, ME, USA).

\section{Subcutaneous orthotopic colorectal cancer transplant model and medical treatment}

The HT-29 colorectal cancer cell line with green fluorescence was established. ${ }^{7}$ BALB/c nude mice, 20 male and 20 female, that ranged from 4- to 6-weeks-old were fed in a special pathogenic free animal facility. The feed was sterilized using cobalt 60 (Xietong Organism Co, Ltd, Nanjing, People's Republic of China). As described above, the subcutaneous orthotopic colorectal cancer transplant model was established successfully. ${ }^{7}$

At 2 weeks postsurgery, 39 out of the 40 nude mice presented with tumors. Based on the volume of the tumors, the 39 mice with tumors were divided into four groups: a control group $(\mathrm{n}=9)$; a group that received $5 \mathrm{mg} / \mathrm{kg}$ of EGCG ( $\mathrm{n}=10)$; a group that received $10 \mathrm{mg} / \mathrm{kg}$ of EGCG $(\mathrm{n}=10)$; and a group that received $20 \mathrm{mg} / \mathrm{kg}$ of EGCG $(n=10)$. In the therapeutic groups, EGCG was administrated intragastrically, and in the control group, $100 \mathrm{uL}$ of physiological saline was administrated intragastrically, once daily for 14 days.

After the treatment of the mice with EGCG for 4 weeks, the growth and metastasis of the primary tumors were continuously monitored using a fluorescent imaging system. After 4 weeks, the primary tumors were weighed and immediately put into liquid nitrogen $\left(-196^{\circ} \mathrm{C}\right)$ and 2 to 3 hours later, these specimens were stored at $-80^{\circ} \mathrm{C}$. In addition, the other parts of the primary tumor and metastases were fixed in $4 \%$ formaldehyde.

\section{TUNEL assay}

Terminal deoxynucleotidyl transferase-mediated dUTPbiotin nick end labeling (TUNEL) assay of tumor slices was performed as reported previously. ${ }^{3}$ For the negative control, all steps were as described above, except that the TUNEL reaction buffer was replaced by phosphate buffered saline (PBS). All slices were evaluated under the microscope. Random $100 \times$ fields of view were selected to count the apoptotic cells and the total number of cells. The apoptotic rate of the tumor cells $=$ apoptotic cells/total cells $\times 100 \%$. 


\section{Real-time polymerase chain reaction (PCR) analysis}

LoVo, SW480, HCT-8, and HT29 cells were treated with $35 \mu \mathrm{g} / \mathrm{mL}$ of EGCG; the expression of Notch 1 , Notch 2, HES1 (hairy-enhancer-of-split 1), and JAG1 (Jagged 1) was studied using the following primers: Notch1: 5'-CAATGTGGATGCCGCAGTTGTG-3' (sense) and 5'-CAGCACCTTGGCGGTCTCGTA-3' (antisense); Notch 2: 5'-AAAAATGGGGCCAACCGAGAC-3' (sense) and 5'-TTCATCCAGAAGGCGCACAA-3' (antisense); HES1: 5'-AGGCGGACATTCTGGAAATG-3' (sense) and 5'-CGGTACTTCCCCAGCACACTT-3' (antisense); JAG1: 5'-CAACCGTGCCAGTGACTATTTCTGC-3' (sense) and 5'-TGTTCCCGTGAAGCCTTTGTTACAG-3' (antisense). Total RNA was extracted as per the instructions provided by Qiagen (Venlo, The Netherlands). The concentration was measured at $260 \mathrm{~nm}$, and the purity of the RNA was obtained by calculating the optical density (OD) ${ }_{260}$ $\mathrm{OD}_{280}$. Real-time PCR analysis was performed using the ABI prism 7300 PCR (Life Technologies, Carlsbad, CA, USA) with SYBR GREEN selected as the fluorescence channel; the internal control was GAPDG (glyceraldehyde-3-phosphate dehydrogenase). The fluorescent signal from the previous 15 cycles was set as the background, and a tenfold standard deviation of the fluorescent signal (between 6 and 15 cycles) was set as the fluorescent threshold value. The cycles that reached these threshold values were referred to as reaching the cycle threshold $(\mathrm{Ct})$. After setting the $\mathrm{Ct}$ and establishing a standard curve, the original concentration was obtained. Finally, the expressions of Notch1, Notch2, HES1, and JAG1 were analyzed.

\section{Western blot analysis}

The primary antibodies used were Abcam Hes1 antibody (ab71559), CST Jagged1 (28H8Rabbit mAb), CST Notch1 (D1E11 XP'M Rabbit mAb), and CST Notch2 (8A1RabbitmAb) (Sigma-Aldrich, St Louis, MO, USA). The secondary antibody was Sigma Goat anti-rabbit HRP. For each of the four cell lines, the cells were seeded in two $90 \mathrm{~mm}$ plates, at a concentration of $5 \times 10^{5}$ cells/plate, with complete medium. After 24 hours, complete medium containing $35 \mathrm{ug} / \mathrm{mL}$ of EGCG was added to one of the two plates containing each cell line, and the cells were cultured at $37^{\circ} \mathrm{C}$ in a humidified atmosphere of $5 \% \mathrm{CO} 2$. Seventy-two hours later, the cells were harvested and washed twice with PBS. The supernatant was discarded and the cells were put on ice. The cells were resuspended in tenfold protein lysis buffer, and the proteins were extracted on ice. The supernatant was obtained after centrifuging at $4^{\circ} \mathrm{C}$ for 20 minutes at $12000 \mathrm{~g}$, and the concentration was measured using the bicinchoninic acid (BCA) assay. Sixty micrograms of total protein were analyzed using $12 \%$ sodium dodecyl sulfate polyacrylamide gel electrophoresis (SDS-PAGE). Then, these proteins

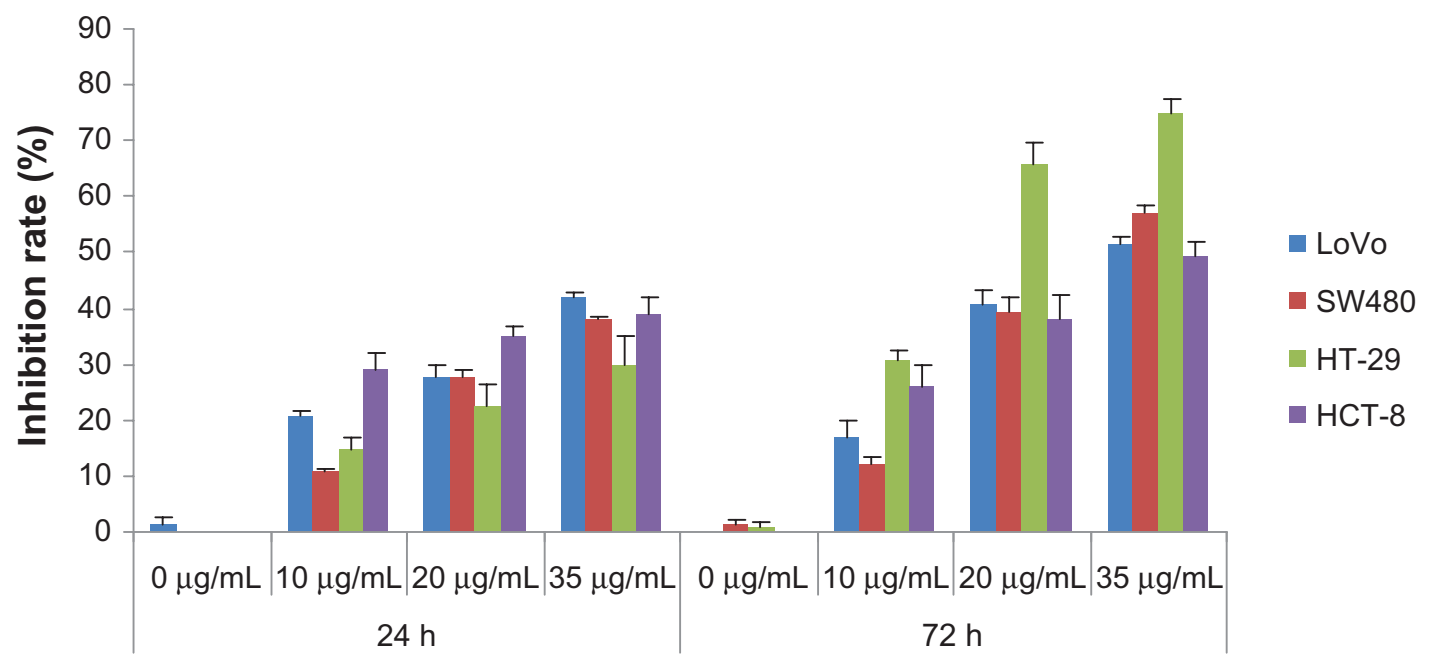

Time after treatment

Figure I The percent EGCG that inhibits the proliferation of colorectal cancer cells at different concentrations.

Notes: The $x$-axis and $y$-axis are the time after treatment and percentage of EGCG that inhibits the proliferation of colorectal cancer cells at different concentrations. Eight experiments were performed for each treatment, and the inhibition rate of cell proliferation was tested for, using a single factor analysis of variance.

Abbreviation: EGCG, epigallocatechin gallate; h, hour. 
were transferred onto a polyvinylidene difluoride (PVDF) film (Sigma-Aldrich) at 20 volts for 40 minutes. The films were blocked using 5\% skimmed milk-TBST (Tris-buffered saline and Tween 20) (Sigma-Aldrich) buffer at $37^{\circ} \mathrm{C}$ for 2 hours, and the proteins were incubated with primary antibodies that were diluted at a ratio of 1:1000. The films were washed three times, and the proteins were incubated with the secondary antibodies that were diluted at a ratio of 1:1000. Finally, these films were washed three times and developed using the enhanced chemiluminescence (ECL) reagent (Sigma-Aldrich). Protein expression was detected using an enhanced chemiluminescence detection system (Thermo Fisher Scientific, Waltham, MA, USA).

\section{Statistical analysis}

These data were analyzed using SPSS 17.0 (IBM, Armonk, NY, USA) and expressed as the mean \pm standard deviation (SD). The inhibition rate of cell proliferation and apoptosis occurring in the cell lines after treatment with EGCG was tested using single-factor analysis of variance (ANOVA). A $P<0.05$ was considered significant. An independentsample $t$-test was used to analyze the mRNA and protein levels of the colorectal cell lines, and a $P<0.05$ was considered significant. A paired-sample $t$-test was used to analyze the tumor proliferation rate, and the expression of proteins in-vivo was considered significant at a $P<0.05$.

\section{Results}

\section{The MTT assay detected the inhibitory} role of EGCG associated with proliferation of the colorectal cancer cells

As shown in Figure 1, increasing the EGCG concentration resulted in an increase in the inhibition of the proliferation of the LoVo cells, SW480 cells, HT29 cells, and HCT-8 cells, at 24 hours and 72 hours. EGCG demonstrated the highest rate of inhibition at the concentration of $35 \mu \mathrm{g} / \mathrm{mL}$. After 24 and 72 hours, the rate of inhibition associated with proliferation increased gradually in all four cell lines. A single-factor ANOVA was used to analyze the inhibition of proliferation at 24 hours and 72 hours, after the cells were treated with different concentrations of EGCG. The results suggest that EGCG inhibited the proliferation of the cells studied when the concentration was increased and that the inhibition was time dependent $(P<0.05)$.

\section{Flow cytometry-detected apoptosis of colorectal cancer cells}

Apoptosis of LoVo cells, SW480 cells, HT29 cells, and HCT- 8 cells significantly increased after the cells were treated with $35 \mu \mathrm{g} / \mathrm{mL}$ ECCG $(P=0.045)$. These findings show that EGCG increased apoptosis of the four colorectal cancer cell lines (Figure 2).

\section{Flow cytometry analysis of the cell cycle}

Flow cytometry was performed using standard techniques, and the data were analyzed with ModFit. Compared with the control group, the LoVo cells at the $\mathrm{G}_{0} / \mathrm{G}_{1}$ phase in the experimental group markedly increased from $18.71 \%$ to $100 \%$. The SW480 cells at the $\mathrm{G}_{0} / \mathrm{G}_{1}$ phase increased from $50.76 \%$ to $58.24 \%$; however, the cells at the $\mathrm{S}$ phase decreased from $48.53 \%$ to $41.76 \%$. The HT2 9 cells significantly increased at the S phase, from $26.08 \%$ to $100 \%$; and the HCT- 8 cells at the $\mathrm{G}_{2} / \mathrm{M}$ phase increased from $7.90 \%$ to $22.90 \%$. These findings indicate that EGCG blocked LoVo cells and SW480 cells at the $\mathrm{G}_{0} / \mathrm{G}_{1}$ phase, HT29 cells at the $\mathrm{S}$ phase, and HCT- 8 cells at the $\mathrm{G}_{2} / \mathrm{M}$ phase (Figure 3 ).

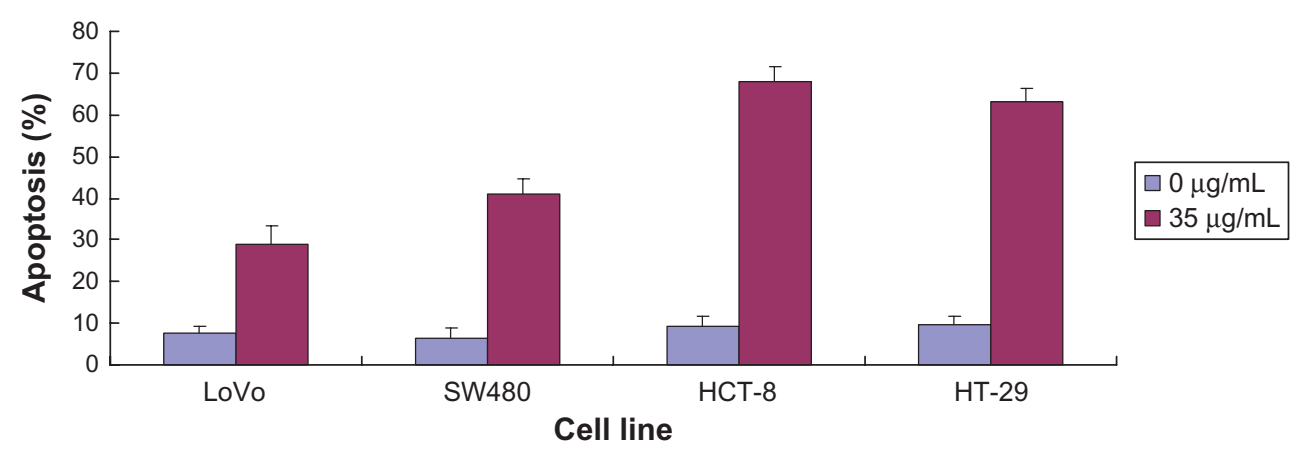

Figure 2 The effect of EGCG on apoptosis of colorectal cancer cells at $0 \mu \mathrm{g} / \mathrm{mL}$ and $35 \mu \mathrm{g} / \mathrm{mL}$.

Notes: The x-axis is the Lovo, SW480, HT-29, and HCT-8 colorectal cell lines. The $y$-axis shows apoptosis of colorectal cell lines after treatment with EGCG. Three experiments were performed for each treatment. Apoptosis of colorectal cancer cells was tested for, using single factor analysis of variance.

Abbreviation: EGCG, epigallocatechin gallate. 

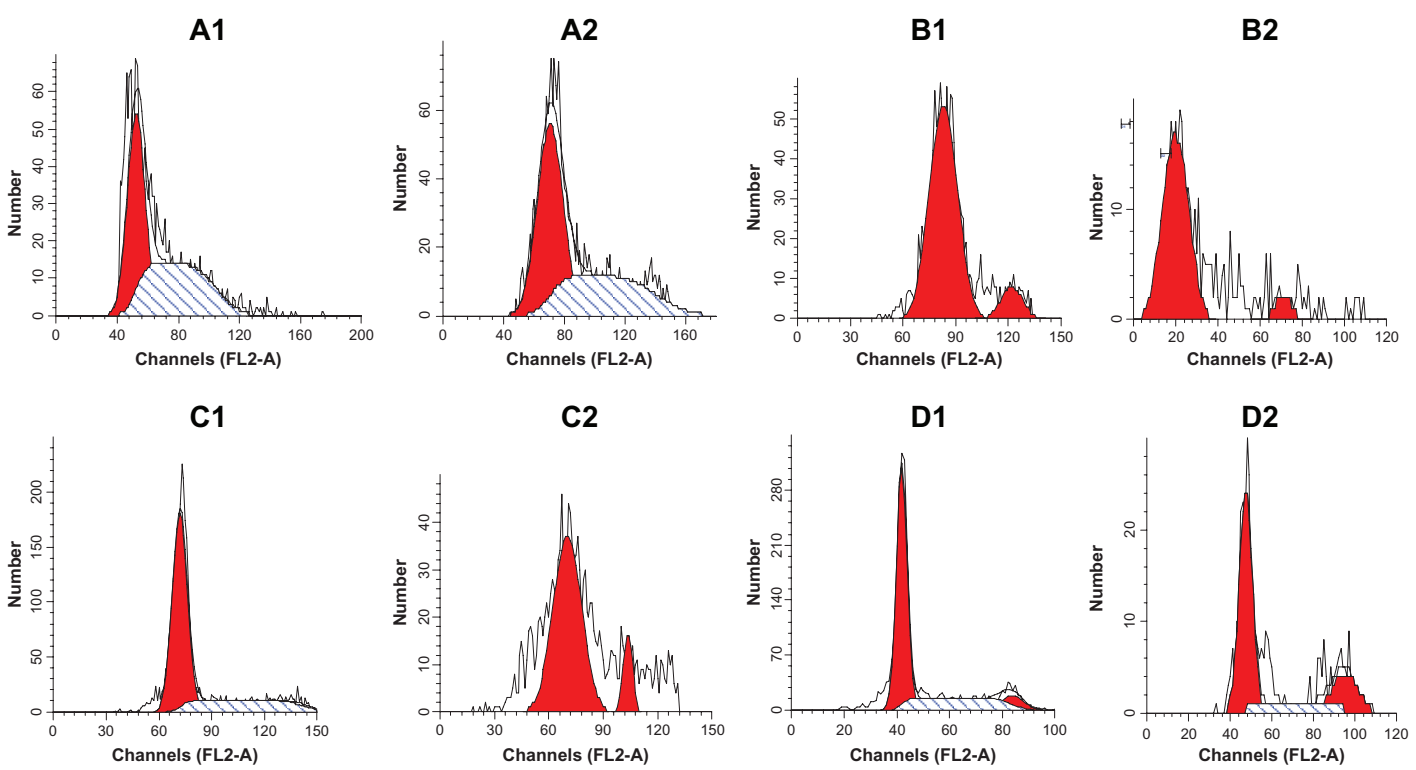

Figure 3 The effect of EGCG on the cell cycle of colorectal cancer cells. (AI) SW480 cells $(0 \mu \mathrm{g} / \mathrm{mL}) ;($ A2) SW480 cells ( $35 \mu \mathrm{g} / \mathrm{mL}) ;($ BI $)$ LoVo cells ( $0 \mu \mathrm{g} / \mathrm{mL}) ;($ B2) LOVO

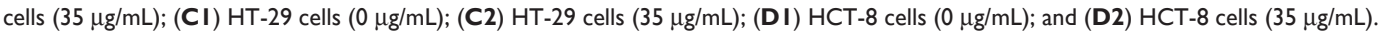

Abbreviation: EGCG, epigallocatechin gallate.

\section{The role of EGCG in the subcutaneous orthotopic transplant model}

The mice were treated with EGCG 14 days after establishing the orthotopic transplant model, and growth curves for the primary tumors were obtained. As shown in Figure 4, the volume of the tumors in the 5,10 , and $20 \mathrm{mg} / \mathrm{kg}$ EGCG groups was reduced on day 7 . On day 14 , the volume of the tumors significantly decreased in the 5 and $20 \mathrm{mg} / \mathrm{kg}$ EGCG groups compared with the control group $(P<0.05)$. On day 28 , the volume of the tumors significantly decreased in the 5 , 10 , and $20 \mathrm{mg} / \mathrm{kg}$ EGCG groups compared with the control group $(P<0.05)$. However, there was no significant difference observed among the three treatment groups $(P>0.05)$.

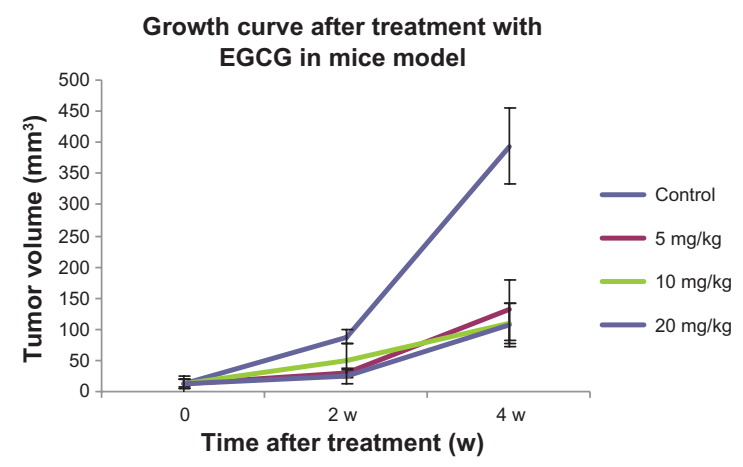

Figure 4 The growth curve for the orthotopic colorectal cancer transplant model treated with EGCG.

Notes: The $x$-axis is the time for the mouse model, after treatment with EGCG, and the $y$-axis is the tumor volume $\left(\mathrm{mm}^{3}\right)$. There were nine mouse models in the control group and ten mouse models for each treatment; a paired-sample $t$-test was used to analyze the tumor proliferation rate.

Abbreviations: EGCG, epigallocatechin gallate; w, weeks.
Lymphatic metastasis was noted in five mice in the control group, in five mice each in the $5 \mathrm{mg} / \mathrm{kg}$ EGCG group and the $20 \mathrm{mg} / \mathrm{kg}$ EGCG groups, and in three mice in the $10 \mathrm{mg} / \mathrm{kg}$ EGCG group. No significant difference was observed among these three groups, and there were no liver or lung metastases.

\section{The TUNEL assay was used to analyze tumor cell apoptosis in the EGCG- treated mice}

The apoptosis-negative nuclei were observed as blue images. The cells positive for apoptosis presented with bigger nuclei that were brown; the apoptotic rates of these tumor cells were calculated. The apoptotic rates of the control group, the $5 \mathrm{mg} / \mathrm{kg}$ group, the $10 \mathrm{mg} / \mathrm{kg}$ group, and the $20 \mathrm{mg} / \mathrm{kg}$ were: $38.04 \%, 51.87 \%, 52.27 \%$, and $54.33 \%$, respectively. The rates of apoptosis in the treatment groups were markedly higher than in the control group $(P<0.05)$. However, there was no significant difference in the rates of apoptosis among the three treatment groups $(P>0.05)$, as illustrated in Table 1.

Table I Apoptosis of tumor cells after the model was treated with $20 \mathrm{mg} / \mathrm{kg}$ of EGCG

\begin{tabular}{llll}
\hline Group & Apoptotic rate & Statistics & P value \\
\hline Control group & $38.04 \pm 12.69$ & & \\
$5 \mathrm{mg} / \mathrm{kg}$ EGCG & $51.87 \pm 10.24$ & -2.495 & 0.024 \\
$10 \mathrm{mg} / \mathrm{kg}$ EGCG & $52.27 \pm 14.06$ & $-2.27 \mathrm{I}$ & 0.036 \\
$20 \mathrm{mg} / \mathrm{kg}$ EGCG & $54.33 \pm 15.54$ & $-2.45 \mathrm{I}$ & 0.026 \\
\hline
\end{tabular}

Abbreviation: EGCG, epigallocatechin gallate. 
A

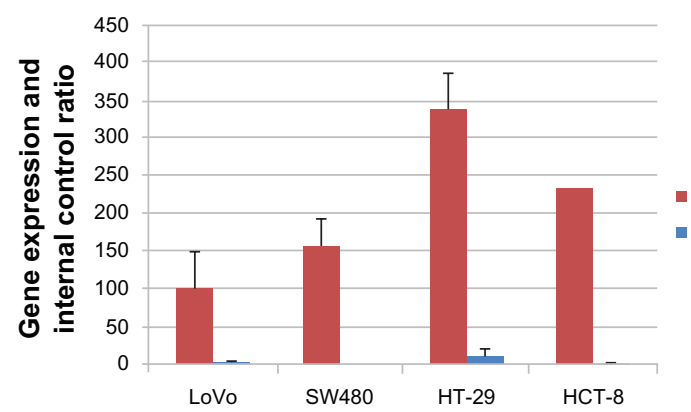

C

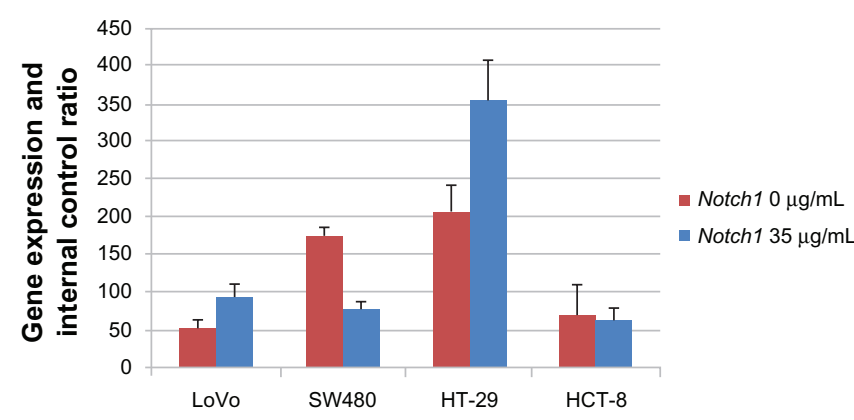

B

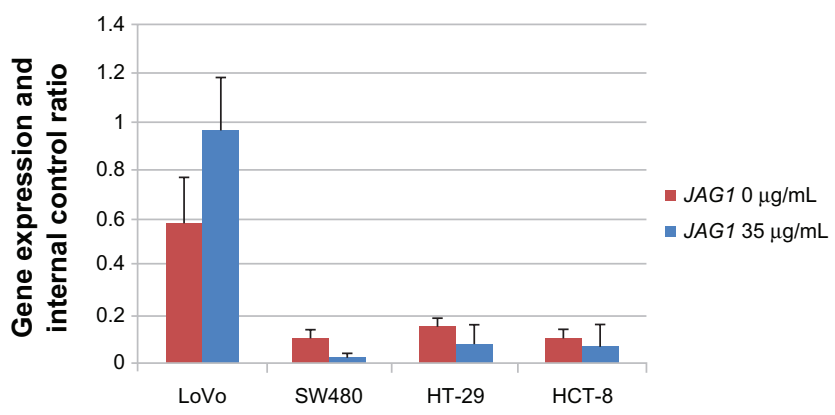

D

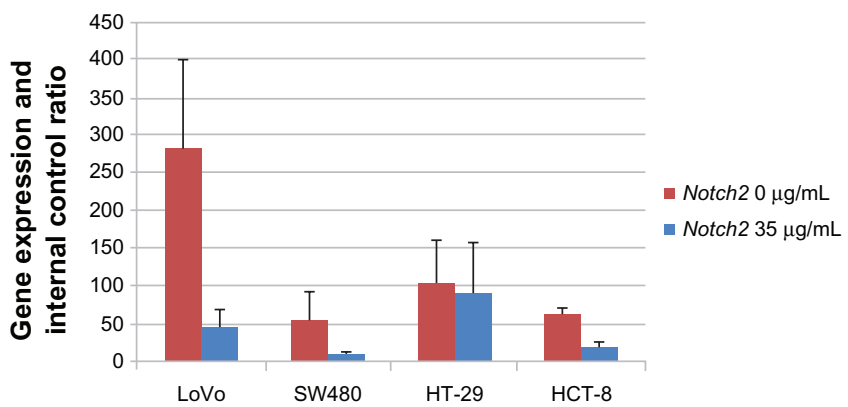

Figure 5 The effect of EGCG on expressions of Notch I, Notch2, HESI, and JAGI in the colorectal cancer cells: (A) the expression of HESI; (B) the expression of JAGI; (C) the expression of Notch I; and (D) the expression of Notch2.

Notes: The x-axis shows the Lovo, SW480, HT-29, and HCT-8 colorectal cell lines. The $y$-axis shows the mRNA of the colorectal cell lines after treatment with EGCG. An independent-sample $t$-test was used to analyze the mRNA levels of the colorectal cell lines.

Abbreviation: EGCG, epigallocatechin gallate.

\section{Gene expression of Notch signaling in the $35 \mu \mathrm{g} / \mathrm{mL}$ EGCG-treated} colorectal cancer cells at the mRNA level

The expression of HESI in the cell lines treated with $35 \mu \mathrm{g} / \mathrm{mL}$ EGCG was significantly lower than in the control group $(P<0.05)$. The expression of $J A G 1$ significantly decreased in the SW480 cells $(P<0.05)$. The expression of Notch 1 was upregulated in the LoVo cells $(P<0.05)$, SW480 cells $(P<0.05)$, HT-29 cells $(P>0.05)$, and the HCT-8 cells $(P>0.05)$. The expression of Notch 2 decreased in the LoVo cells $(P<0.05)$, SW480 cells $(P<0.05)$, and the HCT- 8 cells $(P<0.05)$ (Figure 5).

\section{Gene expression of Notch signaling, at the protein level, in $35 \mu \mathrm{g} / \mathrm{mL}$ EGCG-treated colorectal cancer cells}

As shown in Figure 6, the expression of HES1 in the cell lines that were treated with EGCG was significantly lower than in the control group $(P<0.05)$. The expression
A

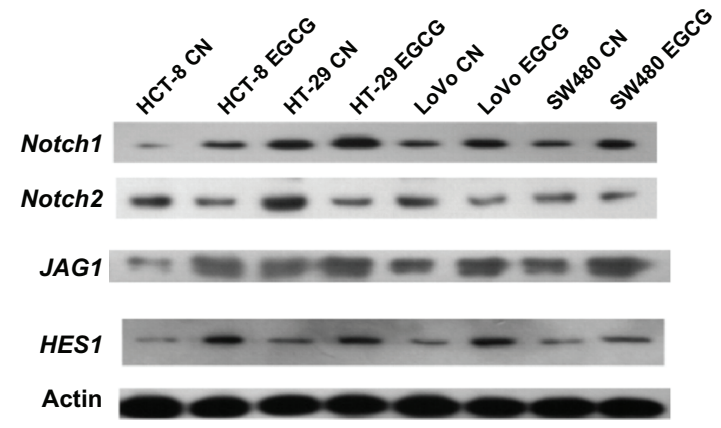

B

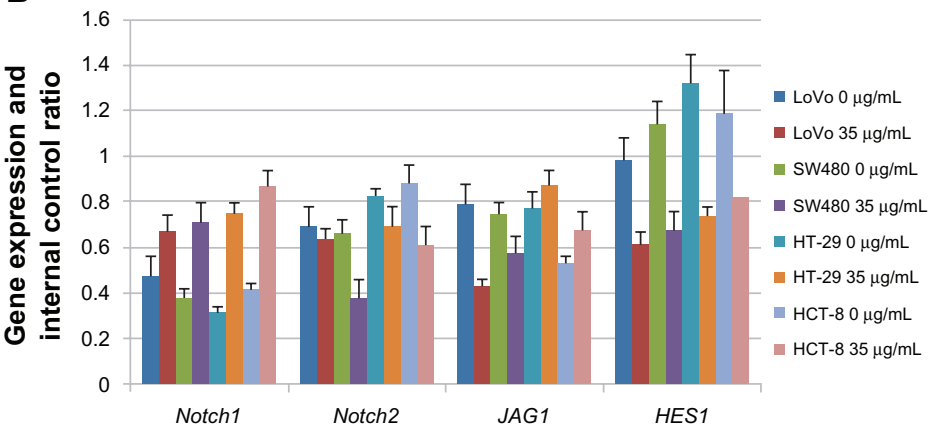

Figure 6 The effect of EGCG on protein expression of colorectal cancer cells: (A) original image; (B) amount of protein expression.

Notes: The $x$-axis shows the Lovo, SW480, HT-29, and HCT-8 colorectal cell lines. The $y$-axis shows the protein level of the colorectal cell lines after treatment with EGCG. An independent-sample $t$-test was used to analyze the protein level of colorectal cell lines.

Abbreviations: EGCG, epigallocatechin gallate; $\mathrm{CN}$, control group. 
of Notch 2 decreased in the SW480 cells $(P<0.05)$. The expression of $J A G 1$ significantly decreased in the SW480 cells $(P<0.05)$. The expression of Notch1 was upregulated in the LoVo cells $(P>0.05)$, SW480 cells $(P>0.05)$, HT-29 cells $(P<0.05)$, and the HCT-8 cells $(P<0.05)$.

\section{Discussion}

Recently, many animal experiments and cell culture studies have suggested that EGCG plays an important role as an antitumor agent that inhibits the development and formation of many tumors. ${ }^{4-6}$ However, its antitumor mechanism remains largely unknown; it may be related to the induction of tumor cell apoptosis, retardation of the cell cycle, ${ }^{8-10}$ and/or the expression of related proteins. ${ }^{4,11}$ Notch signaling plays an important role in the renewal and differentiation of colorectal cancer stem cells. Reports on the genes associated with Notch signaling, such as Notch1, Notch2, HES1, and $J A G 1$, regulated by EGCG have been limited. In this study, the EGCG mechanism of action was explored by analyzing the expression of Notch signaling-related gene activity at the mRNA and protein levels.

The MTT assay demonstrated that EGCG inhibited the proliferation of colorectal cancer cells; this inhibition increased with increasing concentrations and in a time-dependent way. This finding is consistent with the reports of a previous study. ${ }^{4}$ To confirm the role of EGCG in colorectal cancer in-vivo, a subcutaneous orthotopic colorectal cancer cell transplant model was successfully established in mice. ${ }^{7}$ Compared with the control group, different doses of EGCG inhibited colorectal cancer. These findings confirmed that EGCG inhibited the proliferation of colorectal cancer in-vivo. However, no significant difference was observed among the treatment groups. This was assumed to be due to the small difference in doses among the groups. In addition, no significant difference was observed with regard to lymphatic metastasis among the three treatment groups; this was assumed to be due to the high metastatic potential of the HT-29 cells.

The results of this study suggest that EGCG induced apoptosis in cultures of colorectal cancer cell lines. In addition, the rate of apoptosis was positively correlated with the dose of EGCG. EGCG blocked the LoVo and SW480 cells at the $\mathrm{G}_{0} / \mathrm{G}_{1}$ phase, HT29 cells at the $\mathrm{S}$ phase, and HCT- 8 cells at the $\mathrm{G}_{2} / \mathrm{M}$ phase. The TUNEL assay was used to evaluate apoptosis in tissues, in-vivo. The rate of apoptosis in the tumor cells in the treatment groups was significantly higher than the rates in the control group. However, there was no significant difference in the apoptotic rate observed among the treatment groups; this was assumed to be due to the small difference in the treatment dose.

A previous study reported that the expression of HESI and $J A G 1$, the target genes of Notch signaling, was downregulated after the colon cancer cells were treated with tea polyphenol. These findings suggest that a component of the tea polyphenol played a role in regulating Notch signaling. ${ }^{3}$ Normal Notch signaling was important for the proliferation and differentiation of cells as well as the renewal of organs and tissues. Abnormal Notch signaling increased cell proliferation and thus, promoted carcinogenesis. Notch1, Notch2, HES1, and JAG1 were target genes of Notch signaling. ${ }^{12}$ The findings of this study showed that the expression of HES1 in the EGCG treatment group was significantly lower than in the control group; this indicated that EGCG inhibited colorectal cancer cells by downregulating the expression of HES1. The findings of a previous study suggested that HES1 is overexpressed in colorectal cancer cells and is an important risk factor for the prognosis of colorectal cancer. ${ }^{13}$

The results of the current study showed that HES1 was an important target gene of colorectal cancer stem cells. Zhang et $\mathrm{a}^{14}$ found that invasion associated with osteosarcoma decreased by regulating the expression of HES1; this indicated that HES1 plays a role in inhibiting upstream genes. This study is the first to find that EGCG is a biological agent that could significantly inhibit the expression of HES1. The mechanism observed is important in the search for biological agents that might play an important role in cancer suppression. EGCG was found to be associated with colorectal cancer inhibition at the colorectal stem cell level. Notch2 is a receptor of Notch signaling, and its expression has been found to be negatively related to the prognosis of colorectal cancer. ${ }^{13}$ In this study, the expression of Notch 2 decreased in EGCG-treated colorectal cancer cells; this indicated that EGCG inhibited colorectal cancer cells by inhibiting the expression of Notch2. Chen et $\mathrm{al}^{15}$ reported that the downregulation of Notch1 and Notch 2 inhibited the growth of glioblastoma; however, Notch 2 played a more significant role. Chu et a ${ }^{16}$ reported that the downregulation of Notch 2 played an important role in suppression of cancer during the development of colorectal cancer. In this study, the expression of Notch 2 at the mRNA and protein levels decreased during cancer suppression with EGCG treatment, consistent with the findings of Chen et al and Chu et al. 
The expression of $J A G 1$ significantly decreased in the SW480 cells $(P=0.019)$, HT-29 cells $(P>0.05)$, and HCT-8 cells $(P>0.05)$. However, the expression of $J A G 1$ increased in the LoVo cells $(P>0.05)$. The expression of Notchl was upregulated in the LoVo cells $(P<0.05)$, SW480 cells $(P<0.05)$, HT-29 cells $(P>0.05)$, and HCT-8 cells $(P>0.05)$. These data suggest that $J A G 1$ and Notch1 played different roles in different colorectal cancer cell lines; however, these findings require further clarification. Reedijk et al ${ }^{17}$ found that Notch1, LFNG, and HES1 were overexpressed in tumors, by microarray analysis.

Recently, Notch signaling was found to play a role in the tumorigenesis of many tumors by affecting the differentiation of tumor stem cells. It was found that Notch signaling controlled and regulated the differentiation and proliferation of stem cells and frequently changed the gene expression of Notch signaling, which promoted the transformation of stem cells to epithelial cells. ${ }^{18}$ It was also found that the genes associated with Notch signaling were overexpressed in colorectal cancer; this suggests that Notch signaling played a suppressive role in the formation of colorectal cancer. ${ }^{19}$ The results of the present study found that EGCG inhibited colorectal cancer cells by inhibiting the expressions of Notch 2 and HES1. However, the study did not determine whether EGCG first inhibited Notch2 expression and then inhibited HES1 expression or simultaneously inhibited both Notch 2 and HES1. Future studies are needed to determine the differences in the expression of Notchl and JAG1 in different cells.

In conclusion, the findings of the in-vitro and in-vivo experiments performed in this study suggest that EGCG inhibited the proliferation of colorectal cancer cells, induced apoptosis, and influenced the cell cycle. The expression of HES1 and Notch2 was inhibited in the EGCG-treated cell lines; these findings suggest that EGCG inhibited colorectal cancer cells by inhibiting the expression of HES1 and Notch2. The expression of Notch1 and JAG1 changed in different EGCG-treated cell lines; however, the precise mechanism involved requires further study. Notch signaling is important for renewal, differentiation, and proliferation of colorectal cancer stem cells. The findings of the present study suggest a novel approach to the prevention and treatment of colorectal cancer at the stem cell level.

\section{Acknowledgments}

The study is funded by the National Nature Science Foundation of China (No 30572447, 30973837, 81273944).

\section{Disclosure}

The authors report no conflicts of interest in this work.

\section{References}

1. Ahmad N, Feyes DK, Nieminen AL, Agarwal R, Mukhtar H. Green tea constituent epigallocatechin-3-gallate and induction of apoptosis and cell cycle arrest in human carcinoma cells. J Natl Cancer Inst. 1997;89(24):1881-1886.

2. Sadava D, WhitlockE, Kane SE. The green tea polyphenol, epigallocatechin3-gallate inhibits telomerase and induces apoptosis in drug-resistant lung cancer cells. Biochem Biophys Res Commun. 2007; 360(1):233-237.

3. Jin H, Tan X, Liu X, Ding Y. The study of effect of tea polyphenols on microsatellite instability colorectal cancer and its molecular mechanism. Int J Colorectal Dis. 2010;25(12):1407-1415.

4. Sukhthankar M, Alberti S, Baek SJ. (-)-Epigallocatechin-3-gallate (EGCG) post-transcriptionally and post-translationally suppresses the cell proliferative protein TROP2 in human colorectal cancer cells. Anticancer Res. 2010;30(7):2497-2503.

5. Tang SN, Fu J, Shankar S, Srivastava RK. EGCG enhances the therapeutic potential of gemcitabine and CP690550 by inhibiting STAT3 signaling pathway in human pancreatic cancer. PLoS One. 2012;7(2):e31067.

6. Adachi S, Nagao T, To S, et al. (-)-Epigallocatechin gallate causes internalization of the epidermal growth factor receptor in human colon cancer cells. Carcinogenesis. 2008,29(10):1986-1993.

7. Jin H, Yang Z, Wang J, Zhang S, Sun Y, Ding Y. A superficial colon tumor model involving subcutaneous colon translocation and orthotopic transplantation of green fluorescent protein-expressing human colon tumor. Tumour Biol. 2011;32(2):391-397.

8. Ju J, Hong J, Zhou JN, et al. Inhibition of intestinal tumorigenesis in Apcmin/+ mice by (-)-epigallocatechin-3-gallate, the major catechin in green tea. Cancer Res. 2005;65(22):10623-10631.

9. Shimizu M, Deguchi A, Lim JT, Moriwaki H, Kopelovich L, Weinstein IB. (-)-Epigallocatechin gallate and polyphenon E inhibit growth and activation of the epidermal growth factor receptor and human epidermal growth factor receptor-2 signaling pathways in human colon cancer cells. Clin Cancer Res. 2005;11(7):2735-2746.

10. Adachi S, Shimizu M, Shirakami Y, et al. (-)-Epigallocatechin gallate downregulates EGF receptor via phosphorylation at Ser1046/1047 by p38 MAPK in colon cancer cells. Carcinogenesis. 2009;30(9): $1544-1552$.

11. Shimizu M, Shirakami Y, Sakai H, et al. (-)-Epigallocatechin gallate inhibits growth and activation of the VEGF/VEGFR axis in human colorectal cancer cells. Chem Biol Interact. 2010;185(3):247-252.

12. Maillard I, Pear WS. Notch and cancer: best to avoid the ups and downs. Cancer Cell. 2003;3(3):203-205.

13. Jin HY, Zhang HY, Wang X, Xu J, Ding Y. Expression and clinical significance of Notch signaling genes in colorectal cancer. Tumour Biology. 2012;33(3):817-824.

14. Zhang P, Yang Y, Nolo R, Zweidler-McKay PA, Hughes DP. Regulation of NOTCH signaling by reciprocal inhibition of HES1 and Deltex 1 and its role in osteosarcoma invasiveness. Oncogene. 2010;29(20): 2916-2926.

15. Chen J, Kesari S, Rooney C, et al. Inhibition of notch signaling blocks growth of glioblastoma cell lines and tumor neurospheres. Genes Cancer. 2010;1(8):822-835.

16. Chu D, Zheng J, Wang W, et al. Notch2 expression is decreased in colorectal cancer and related to tumor differentiation status. Ann Surg Oncol. 2009;16(12):3259-3266.

17. Reedijk M, Odorcic S, Zhang H, et al. Activation of Notch signaling in human colon adenocarcinoma. Int J Oncol. 2008;33(6):1223-1229.

18. Radtke F, Raj K. The role of NOTCH in tumorigenesis: oncogene or tumour suppressor? Nat Rev Cancer. 2003;3(10):756-767.

19. Qiao L, Wong BC. Role of Notch signaling in colorectal cancer. Carcinogenesis. 2009;30(12):1979-1986. 
OncoTargets and Therapy

\section{Publish your work in this journal}

OncoTargets and Therapy is an international, peer-reviewed, open access journal focusing on the pathological basis of all cancers, potential targets for therapy and treatment protocols employed to improve the management of cancer patients. The journal also focuses on the impact of management programs and new therapeutic agents and protocols on

patient perspectives such as quality of life, adherence and satisfaction. The manuscript management system is completely online and includes a very quick and fair peer-review system, which is all easy to use. Visit http://www.dovepress.com/testimonials.php to read real quotes from published authors.

Submit your manuscript here: http://www.dovepress.com/oncotargets-and-therapy-journal 